\title{
Novel Cardiac Progenitors for All Components of the Heart Except for the Right Ventricle
}

\author{
Hiroki Kokubo, Masayuki Fujii, Akane Sakaguchi, \\ Masao Yoshizumi, and Yumiko Saga
}

\begin{abstract}
Mammalian heart is first recognized as the cardiac crescent at the egg cylinder stage, which had been thought to form all components of the heart. Recently, several lineage-tracing experiments using dye-labeling and mouse genetic technique have demonstrated that another cardiac progenitor population, called the second heart field, contributes to the outflow tract, the right ventricle, and a part of the atria. This finding has provided a great impetus for the understanding of cardiac morphogenesis, but it has also raised additional questions such as the potential existence of unique cardiac progenitors that give rise to the left ventricle and the conduction system. Here, we show that a novel cardiac progenitor population is identified by expression and lineage tracing analysis of the Sfrp5 gene. We found that Sfrp5 gene expression was constantly seen in the venous pole of the forming heart, which is later differentiated into the myocardium of the sinus venosus, a precursor of the sinoatrial node. Descendants of Sfrp5expressing cells were found to contribute not only to the sinus venosus, but also to the left ventricle, atria, and the outflow tract by lineage-tracing analysis using Sfrp5-Cre and -Ert2Cre mice. These results indicate that Sfrp5-expressing cells could include progenitors for the sinus venosus, atria, the left ventricle, and the outflow tract, but not in the right ventricle, implying that the origin of the right ventricle essential for pulmonary circulation could be unique from progenitors for other components in mammalian cardiac development. In this session, we would like to discuss a new understanding of cardiac progenitor distribution.
\end{abstract}

H. Kokubo $(\bowtie) \cdot$ M. Fujii $\cdot$ M. Yoshizumi

Department of Cardiovascular Physics Medicine, Hiroshima University, Hiroshima, Japan e-mail: hkokubo@hiroshima-u.ac.jp

A. Sakaguchi · Y. Saga

Determent of Mammalian Development, National Institute of Genetics, Mishima, Japan 


\section{Keywords}

Sfrp5 $\cdot$ Heart development

Mammalian cardiac development starts with the cardiac crescent, which forms the primary heart tube and then the four-chambered heart along with the progressive differentiation of progenitors into cardiomyocytes [1-3]. In this process, two independent pulmonary and systemic circulatory systems are established. For the establishment of these systems, remodeling of the ancestral heart into these two separate systems might occur with the production and rearrangement of progenitor cells. Recent findings pertaining to another cardiac lineage called the secondary heart field (SHF) modified the classical view of cardiac development that the myocardium was derived from a single source. The SHF emerges in the splanchnic mesoderm to supply cells at both the arterial and venous poles of the heart tube, including the right ventricle (RV), most of the atria and outflow tract (OFT). However, the progenitor population that forms the first heart field (FHF), which gives rise to the left ventricle (LV) and the rest of the atria and the sinus venosus (SV) are still ambiguous. Since Wnt signalling is one of the most important regulatory pathways in vertebrate cardiogenesis [4-8], we focused on the Secreted frizzled-related protein (Sfrp) sub-family, known to encode secreted decoy receptors for Wnt signalling, and analyze its expression and lineage during cardiac development [9].

We first observed expression of Sfrp5. At E7.5, Sfrp5 was detected in the lateral and caudal region of the $M l c 2 a$ and $N k x 2-5$ expressing region in the cardiac crescent. This region was distinct from the Isll and Hcn4-expressing region, but mostly overlapped with the region marked by Tbx5. These observations suggest that Sfrp5 expression seems to be restricted in undifferentiated FHF progenitors existing in the lateral sides of the cardiac crescent. During the heart tube looping stage (E8.0 E8.5), Sfrp5 was continuously expressed in the venous pole of the heart. This Sfrp5expressing area was distinct from the expression area of $M l c 2 a$ and $N k x 2-5$, indicating that Sfrp5 is exclusively expressed in the undifferentiated cardiomyocytes. At E9.5, Sfrp5 expression was restricted to the Tbx18 and WT1 expressing the ventral venous pole, suggesting that Sfrp5 is expressed in progenitors of such SV and epicardium.

Since Sfrp5 expression was found in progenitors of the FHF at E7.5, and also detected continuously in the progenitors of the SV and the epicardium, we hypothesize that Sfrp5-expressing cells include progenitors for the LV, the atrium, the SV and the epicardium. To test this possibility, we performed lineage analysis of Sfrp5-expressing cells by crossing the Sfrp5-Cre line with Rosa26R or CAGfloxed-CAT-eGFP reporter mice [10]. We found that $\beta$-galactosidase (LacZ) labeled cells were detected in the OFT, LV, atria, and venous pole, but not in the RV 
at E9.5. Labeled cells overlapped with TnT in the OFT, LV, and atria, suggesting that descendants of Sfrp5-expressing cells differentiated into the chamber myocardium in addition to the SV. At E13.5, LacZ-stained cells were mostly found in the LV and also in the WT1-expressing pericardium and the CD31-expressing endocardium. Labelled cells were found in part of the interventricular septum but rarely in the RV. These observations suggest that Sfrp5 is expressed in progenitors of the $\mathrm{SV}$, pericardium, epicardium, endocardium, and the OFT, as well as in all chamber myocardium except for the RV.

Since Sfrp5 expression is restricted in the venous pole of the heart at E8.5, which does not include the chamber myocardium, we speculated that cells expressing Sfrp5 prior to E8.5 might include progenitors of the FHF. To evaluate the fate of Sfrp5-expressing cells at specific developmental stages, we crossed a tamoxifen inducible Sfrp5- ERT2 Cre mouse line with reporter mice and found that LacZ staining revealed that cells labelled at E7.5 were found in the OFT, LV, atria, SV, and venous pole but not in the RV. Moreover, labelled cells at E8.5 and E9.5 were still found in the atria, indicating that Sfrp5-expressing cells at the venous pole may supply cardiomyocytes for the inflow tract. These data suggest that Sfrp5-expressing cells at E7.5 include common progenitors for the SV, OFT, and all chamber myocardium except for the RV.

Although the expression pattern of Sfrp 5 was distinct from that of Isll, an SHF marker at E7.5, descendants of Sfrp5-expressing cells were found in the OFT, which was thought to be derived from the SHF. This observation gave rise to a hypothesis that the expression of Sfrp5 precedes that of Isll in the splanchnic mesoderm. To test this possibility, we performed lineage-tracing analysis using Isll-Cre and CAGfloxed-CAT-mRFP reporter/Sfrp5-venusYFP mice and found that the YFP signal in the ventral venous pole rarely overlapped with derivatives of Isll-expressing cells. Instead, in Sfrp5-Cre/CAG-floxed-CAT-eGFP-reporter mice, GFP-labelled cells expressed Isll in the dorsal splanchnic mesoderm and part of the OFT. These findings indicate that part of the SHF lineage is derived from Sfrp5-expressing cells to contribute to the OFT and atria.

Based on our findings, we propose a model for the contribution of Sfrp5expressing cardiac precursors to heart development (Fig. 50.1). We found that Sfrp5-expressing cells at E7.5 contributed to the OFT, the LV, the atria, and the $\mathrm{SV}$, but not to the RV, by fate mapping experiments using Sfrp5-Cre and -ERT2Cre mice. This observation indicates the possibility that the FHF lineage and the SV might share a common origin. Given that Isll lineages contribute to all myocardium except for the LV [11, 12], the fate of Isll lineages to the RV or Sfrp5 lineages to the LV may be exclusively determined during the earliest stages of cardiogenesis. 

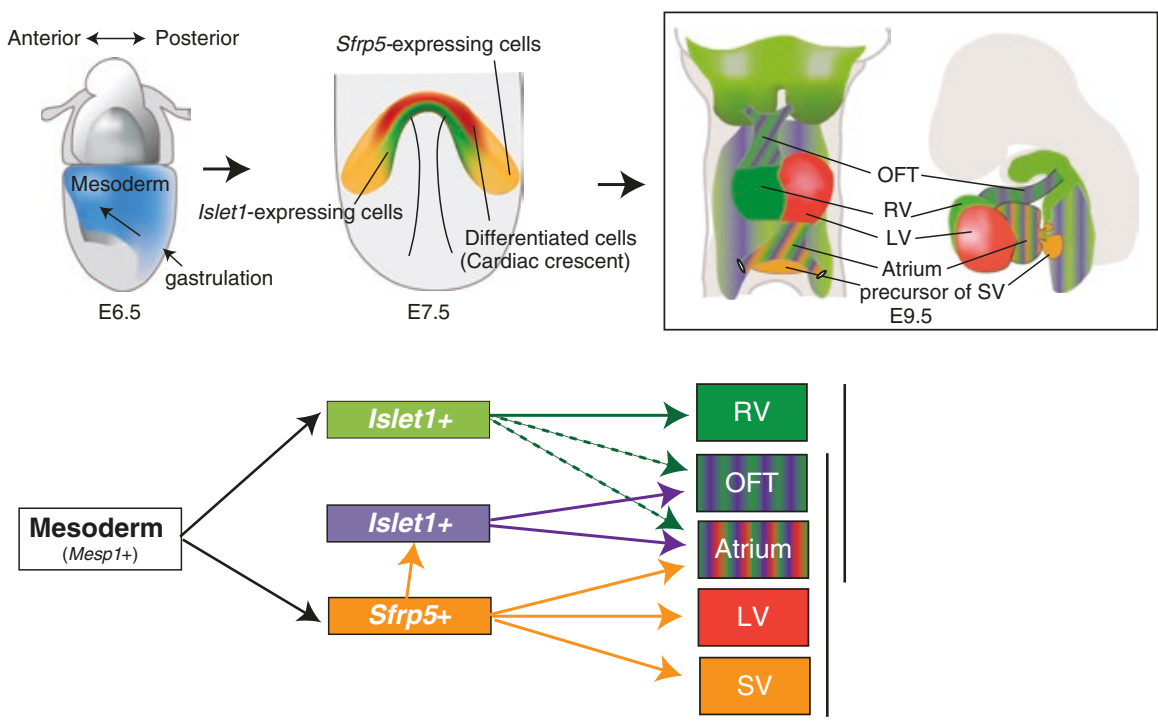

Fig. 50.1 A new model for heart development: Crescent shaped heart primordium is divided into two layers along the mediolateral axis. The medial side of heart crescent, expressing Islet 1, forms splanchnic mesoderm and its decedents contribute to all cardiac components except the LV. On the other hand, the lateral side of heart crescent, expressing Sfrp5, localizes in the mesenchymal cells in the inflow tract to form the $\mathrm{SV}$, and its decedents contribute to all cardiac components except the RV

\section{References}

1. Moorman AF, Christoffels VM, Anderson RH, van den Hoff MJ. The heart-forming fields: one or multiple? Philos Trans R Soc Lond B Biol Sci. 2007;362:1257-65, G5L17245615197K8 [pii]. https://doi.org/10.1098/rstb.2007.2113.

2. Buckingham M, Meilhac S, Zaffran S. Building the mammalian heart from two sources of myocardial cells. Nat Rev Genet. 2005;6:826-35. https://doi.org/10.1038/nrg1710.

3. Abu-Issa R, Kirby ML. Heart field: from mesoderm to heart tube. Annu Rev Cell Dev Biol. 2007;23:45-68. https://doi.org/10.1146/annurev.cellbio.23.090506.123331.

4. Brade T, Manner J, Kuhl M. The role of Wnt signalling in cardiac development and tissue remodelling in the mature heart. Cardiovasc Res. 2006;72:198-209. https://doi.org/10.1016/j. cardiores.2006.06.025.

5. Cohen ED, Tian Y, Morrisey EE. Wnt signaling: an essential regulator of cardiovascular differentiation, morphogenesis and progenitor self-renewal. Development. 2008;135:789-98. https://doi.org/10.1242/dev.016865.

6. Gessert S, Kuhl M. The multiple phases and faces of wnt signaling during cardiac differentiation and development. Circ Res. 2010;107:186-99. https://doi.org/10.1161/ CIRCRESAHA.110.221531.

7. Qyang Y, et al. The renewal and differentiation of Isl1+ cardiovascular progenitors are controlled by a Wnt/beta-catenin pathway. Cell Stem Cell. 2007;1:165-79., 10.1016/j. stem.2007.05.018.

8. Ueno $\mathrm{S}$, et al. Biphasic role for Wnt/beta-catenin signaling in cardiac specification in zebrafish and embryonic stem cells. Proc Natl Acad Sci U S A. 2007;104:9685-90. https://doi. org/10.1073/pnas.0702859104. 
9. Fujii M, et al. Sfrp5 identifies murine cardiac progenitors for all myocardial structures except for the right ventricle. Nat Commun. 2017;8:14664. https://doi.org/10.1038/ncomms14664.

10. Soriano P. Generalized lacZ expression with the ROSA26 Cre reporter strain. Nat Genet. 1999;21:70-1. https://doi.org/10.1038/5007.

11. Moretti A, et al. Multipotent embryonic isl1+ progenitor cells lead to cardiac, smooth muscle, and endothelial cell diversification. Cell. 2006;127:1151-65. https://doi.org/10.1016/j. cell.2006.10.029.

12. Laugwitz KL, Moretti A, Caron L, Nakano A, Chien KR. Islet1 cardiovascular progenitors: a single source for heart lineages? Development. 2008;135:193-205. https://doi.org/10.1242/ dev.001883.

Open Access This chapter is licensed under the terms of the Creative Commons Attribution 4.0 International License (http://creativecommons.org/licenses/by/4.0/), which permits use, sharing, adaptation, distribution and reproduction in any medium or format, as long as you give appropriate credit to the original author(s) and the source, provide a link to the Creative Commons license and indicate if changes were made.

The images or other third party material in this chapter are included in the chapter's Creative Commons license, unless indicated otherwise in a credit line to the material. If material is not included in the chapter's Creative Commons license and your intended use is not permitted by statutory regulation or exceeds the permitted use, you will need to obtain permission directly from the copyright holder. 